\title{
Performing Transactions Simultaneously in Multiple Heterogeneous Database Instances using Vocal Commands with One Time Password Authenticator as an Extended Security Feature
}

\author{
Vignesh $\mathrm{J}$ \\ M.Tech (CSE) \\ SASTRA University \\ Tamilnadu, INDIA 613401.
}

\author{
Dr.A.Umamakeswari, \\ Associate Dean - SOC \\ SASTRA University \\ Tamilnadu, INDIA 613401.
}

\begin{abstract}
Now-a-days databases have become an integral part of human life. Every human maintains his/her data in some form, which he/she understands easily. Multiple Heterogeneous databases are used whose operations will be handled by using speaker vocals, i.e. speaker will issue a vocal command and the databases will act/respond so. The main aim is to make use of voice (i.e.) vocal tone to operate multiple databases, thereby providing complete hands-free human and database interaction. Multiple database concept acts as a lifeline by providing protection against database crashes, data loss, etc...(Say if one database containing all the essential data crashes (or) corrupts the other database comes into the rescue and the data's can be retrieved from it). The complete access is also prohibited to intruders by providing one time password as an extended security feature.
\end{abstract}

Index Terms: - Multiple Databases, Speaker Vocals, Database Crashes, Data Loss, Onetime Password, SR Systems

\section{INTRODUCTION}

Two decades back computer and its operations remained mystery to many but now-a-days it is not so. In today's world we are not only able to perform almost all operations in an easy way with the aid of computers but also in a highly interactive user friendly manner. Other than the recent trends like touch screen which made human-computer interaction easy, we also have application specific software such as speech to text converters (which also makes HCI easier). Speech recognition (SR) systems allow people to control a computer by speaking to it through a microphone, either entering text, or issuing commands to the computer.

Databases have become integral part of human life. Multiple databases facilitate creation of instances of different databases and handle them efficiently and effectively in timely manner. It is highly important that all operations in a multiple database are carried out by using speaker vocals using One Time Password for user authentication provided as an extended security feature.

Two types of Speech Technologies are available:

1. Discrete Speech - The early speech recognition programs all used discrete speech, which forced the user to have a short pause between words as they were dictated. 2. Continuous Speech - Systems using Continuous speech, expects user to dictate complete phrases or sentences in one go. People who can benefit from the use of Speech Recognition (SR) Systems are People with Physical Access Difficulties, People with conditions such as RSI (Repetitive Strain Injury), arthritis, high spinal injury and People with Specific Learning Difficulties (Dyslexia). SR systems use words from its own dictionary and the spelling will generally be correct. Unfortunately, a word guessed by the SR system will not necessarily be the same as the one spoken by the user. User needs to have sufficient word recognition skills to be able to choose the desired word from the list of choices or enter it $\mathrm{him} /$ herself. The dynamic prediction feature is very useful since the desired word will usually appear quickly provided that the user correctly guesses the first 2 or 3 letters. [3]

\section{OBJECTIVE}

Establishing connectivity among multiple heterogeneous databases and operating it completely through human vocals, thereby achieving a complete hands-free "Human-Database interactivity". All database operations such as insertion, deletion and updation of records are to be performed through human vocal commands. Records should be simultaneously inserted, into, deleted and also updated from multiple heterogeneous databases instances available at different locations.

\section{PROBLEM STATEMENT}

\subsection{Existing System}

The following are the study made with respect to databases: Telephony voice operated database exists: Datas are collected from individuals through telephone and are manually fed into databases. [3]

EMR (Electronic Medical Record) exists: Datas pronounced through voice are stored in word format file; datas may be patient record, e.t.c. [4]

Direct voice operated multiple Heterogeneous databases instances don't exist: Simultaneous transactions in SQL Server database and MS Access database, operated using human vocals does not exist.

\subsection{Proposed System}

The need of the day is to have a complete voice operated multiple databases designed with enhanced security. For example, SQL Server database and MS-Access database simultaneously operable through human vocals shall be designed, thereby preventing data loss, database crashes. The design is implemented as Separate Software Package installable on all windows machine. 


\section{BASIC ARCHITECTURE OF THE PROPOSED SYSTEM}

The architectural diagram in Fig. 1 shows that the USER is responsible for operating all entities through MIC using his / her vocal tone, the entities here include PC, DATABASES, FRONTEND. The USER uses PROGRAMMING LANGUAGE(PGM LANG) to design the FRONTEND and to connect DATABASES with the FRONTEND. The design will be highly interactive as even the PC speaks out for inputs whereever necessary. The entire design also performs necessary validation whenever required.

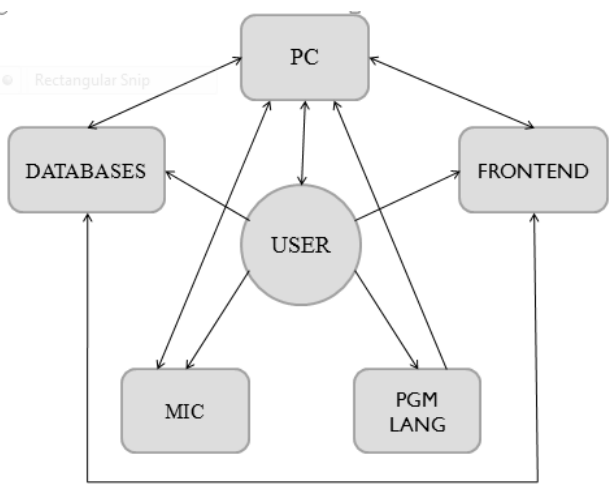

Fig.1: Architectural Diagram

The design also prevents unauthorized access to the Multiple Databases by providing one time password as an Authentication function for each transaction.

\section{CLASS DIAGRAM}

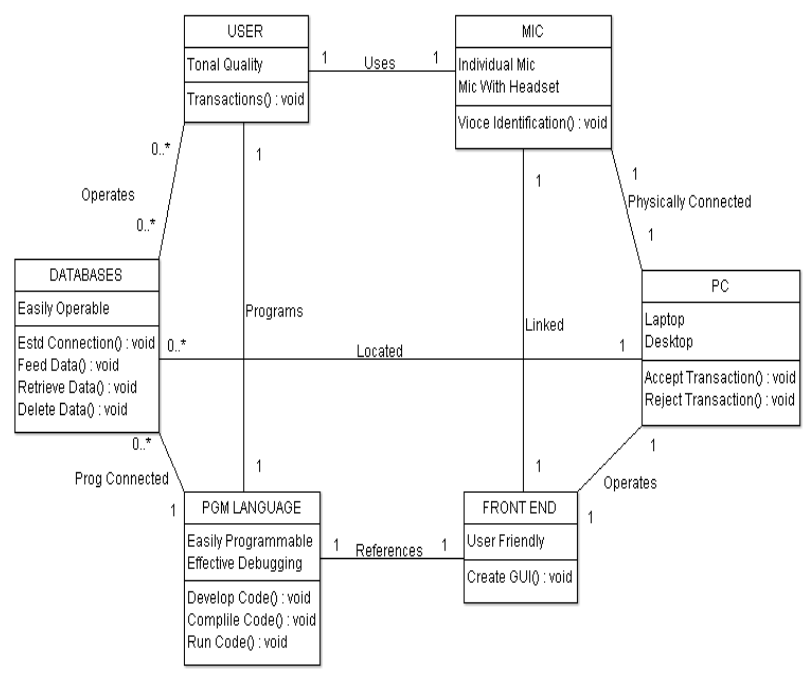

Fig. 2: Class Diagram

Fig. 2 depicts the overall workflow of the voice operated multiple heterogeneous databases. The entities involved are:User, MIC, Databases, Programming Language, Front End (GUI), $P C$. The User uses Programming Language to create Front End and also uses it for establishing connectivity among Databases. A MIC that accepts user vocal inputs is used. When User utters a word through MIC, the word should be correctly recognised and used appropriately to perform certain Database operations, the User pronounces the vocal commands through MIC and uses Programming Language to operate upon Databases.

\section{FATAL 4 WAY COMMUNICATION SCHEME}

The Fatal 4 way communication scheme in fig. 3 specifies the four entities among which there should exist a reliable communication. The entities User, Computer (PC), Front End (GUI), Backend (Databases) should effectively communicate among each other to make a transaction successful. There should exist a time specific communication. Communication between USER and FRONTEND is very essential as the FRONTEND is responsible for GUI, and ultimately responsible for storing datas in MULTIPLE DATABASES (BACKEND) ,i.e. fields available in GUI where datas are entered using human vocals through a MIC.

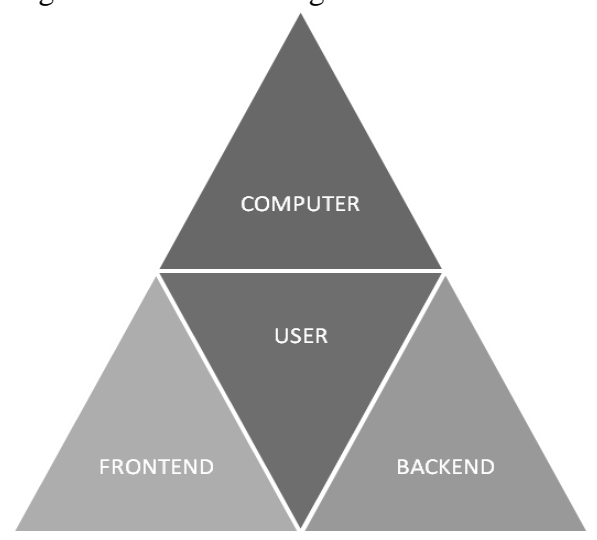

Fig. 3: Four way communication scheme

The entire operation involved in the design will be completely operated through human vocals thereby achieving complete Hands Free Human-Database Interaction

\section{DEVELOPMENT PHASES}

7.1 Initializing Speech:

Basic Training for System to Accept Vocals:

The System (PC-Laptop/Desktop) must be sufficiently trained to accept the human spoken words, letters, numbers and special characters. [1]

Making System to respond to human vocals:

The System should not be idle when user utters words, numbers, letters, and special characters and must provide response by triggering certain event, or should throw some messages indicating the acceptance or rejection of user vocals.

Enabling System to Speak-out:

Two way communications should be encouraged, neglecting the age old one way communication, where only users communicate to systems. On making system to speak-out to users, validations can be made effective.

\subsection{One Time Password Generation as an Extended Security Feature}

Now-a- day's security plays an essential role in assessing an application/product. If security is adequate then the application/product will have a fair run in the market and if the security is compromised or not adequate then the vice versa will happen. Hence the idea here is to develop a one time password generator which will keep on generating a new password for each new transaction and which will provide authentication only when the one time password is used in conjunction with user credentials, thereby enhancing security 
to certain extent. One Time Password provides protection against Data Theft.

\subsection{Multiple Heterogeneous Databases Instance Creation}

Heterogeneous database Instances must be created. Suppose an instance of SQL Server is made available at a

Location, an instance of MS-Access or some other database instance must be made available at different location, such that data losses and data corruption can be prevented. Instances of Databases created and deployed at different locations must be password protected so as to prevent unauthorized access to database instances.

\subsection{Establishing Multiple Database Connectivity}

Once the Heterogeneous database instances are created, connectivity among the multiple database instances and front end or the GUI must be established. Connectivity established should adhere to the following scheme:-

Record Insertion to particular database instance: Information entered into fields of the GUI, should be inserted as a record into the single database instance available at a particular location.

Simultaneous Record Insertion to multiple database instances: Records must be inserted into multiple heterogeneous database instances available at different locations simultaneously.

Record Deletion from a particular database instance: Record deletion from particular database instance available at a particular location.

Simultaneous Record Deletion from multiple database instances: Record deletion from multiple heterogeneous database instances available at different Locations.

\subsection{SCREENSHOTS}

\subsubsection{Initial Screen}

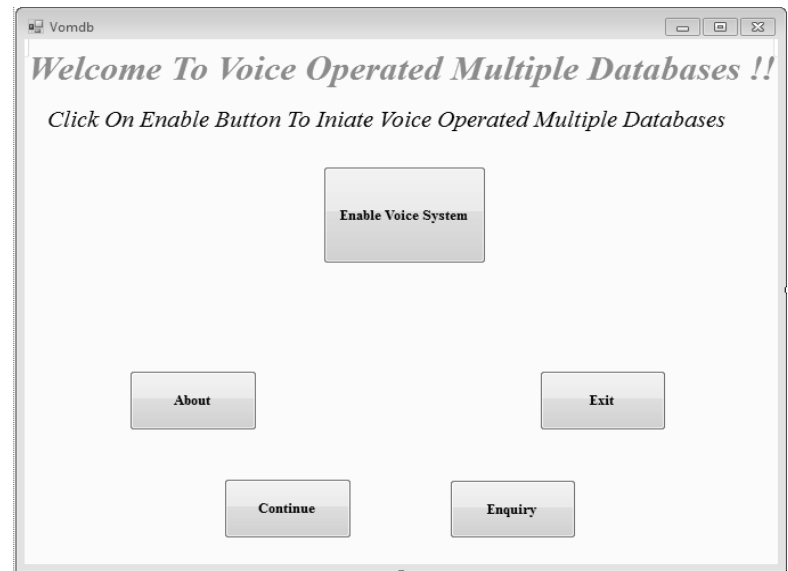

Initial Screen is the start-up Page where the user can start the Speech Engine through a mouse click on the button Enable Voice System, once the button Enable voice System is clicked the speech engine gets activated and the whole system is ready to accept human vocal commands, the other buttons available are 1.About: which speaks out the details regarding system Such as Insertion, Deletion, Updation of records through his/her vocals.

\subsubsection{Screenshot for Insertion into Access} Database Instance at One Location functions and also about the authors of this system , 2.Enquiry: speaks out details wherein the end users of system can contact the authors for their queries, 3.Exit: is used to close the complete application and 4.Continue: button takes the users to next screen wherein the users are allowed to carry out their transactions.

\subsubsection{One Time Password Authenticator}

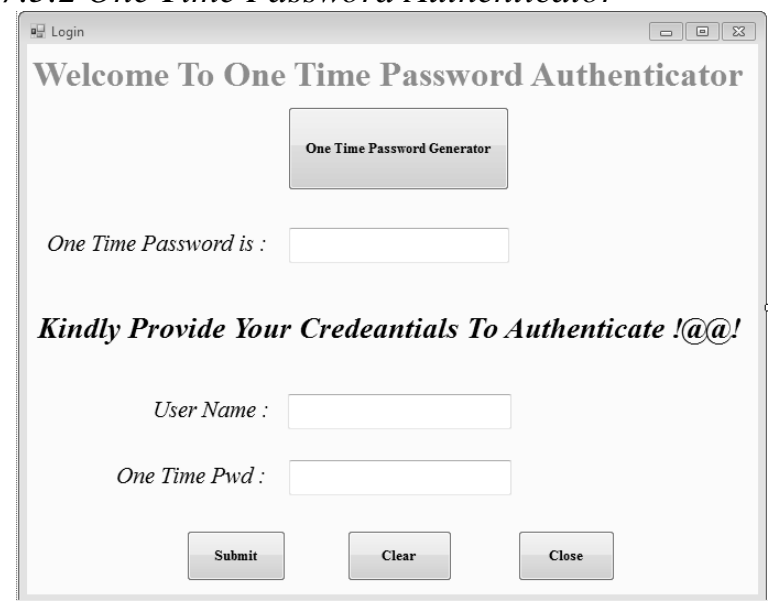

The one time password generator is the second screen which ensures the authenticity of the application. This function keeps on generating dynamic one time passwords that serves the user authentication, the onetime password should be used in conjunction with the user name to perform user login. If wrong user credentials are provided the application closes automatically.

\subsubsection{Multiple Heterogeneous Database Instances} GUI

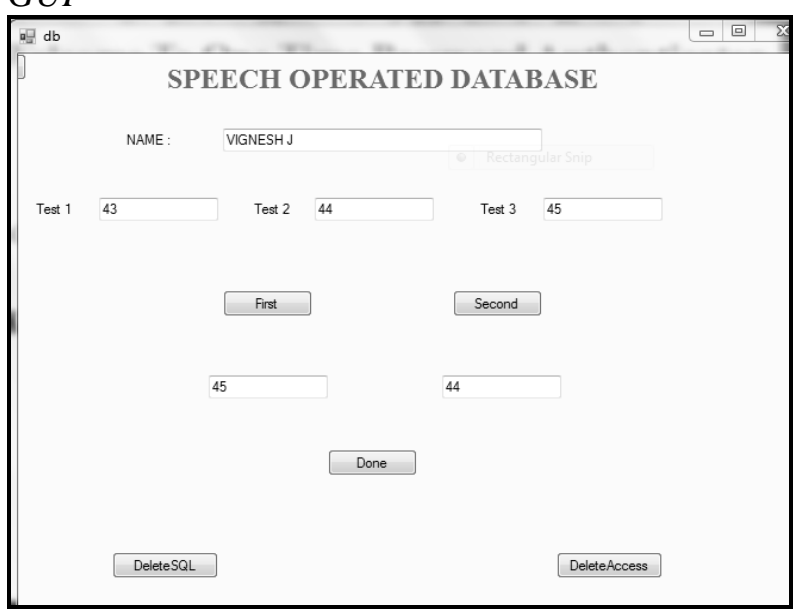

Multiple Heterogeneous Databases GUI is the screen where actual user transaction is done, the user through vocal commands inserts the necessary inputs in the fields provided and performs necessary database operations

\begin{tabular}{|l|llll|}
\hline & NAME & TEST1 & TEST2 & TEST3 \\
\hline 7 & ravi & 34 & 43 & 44 \\
& VIGNESH J & 43 & 44 & 45 \\
\hline & NULL & NULL & NULL & NULL \\
\hline
\end{tabular}


7.5.5 Screenshot for Insertion into SQL Server Database Instance at another Location

\begin{tabular}{|l|llll|}
\hline & NAME & TEST1 & TEST2 & TEST3 \\
\hline * & VIGNESH J & 43 & 44 & 45 \\
$*$ & NULL & NULL & NULL & NULL \\
\hline
\end{tabular}

\section{CONCLUSIONS}

Hence the use of voice (i.e.) vocal tone to navigate and operate Multiple Heterogeneous Databases thereby providing a complete hands-free human-computer interaction, achieves efficient results. This facilitates the physically challenged users to use computer application like normal users. Discrete speech technology has been used for performing operation on buttons and continuous speech technology has been used for entering datas into fields. Voice Operated Multiple Heterogeneous Databases comes into rescue when the datas in one database instance has corrupted or lost, since we have made use of multiple heterogeneous databases available at different locations i.e. if datas from one database instance cannot be retrieved, the similar datas can be retrieved from the other database instance.

\section{REFERENCES}

1. B. Balentine, D. Morgan, and W. Meisel, How to Build a Speech Recognition application, Enterprise Integration Group, 1999.

2. Microsoft Speech SDK, Version 5.1 Documentation, Microsoft Corporation, 2001.

3. Direct Voice Control Speech Data Entry and Database Query Models, LINDI 2007 , International Symposium on Logistics and Industrial Informatics,13-15 September, 2007 Wildau, Germany.

4. A Voice-enabled, Structured Medical Reporting SystemDavid F. Rosenthal, PhD, JoAnne M. Bos, MS, Rachael A. Sokolowski, Jennifer B. Mayo, Kerry A. Quigley, Roger A. Powell, MA, and Mary-Marshall Teel. 